\title{
A NOTE ON EQUIVARIANT ETA INVARIANTS
}

\author{
ZHANG WEIPING
}

(Communicated by J. Marshall Ash)

\begin{abstract}
We prove the regulairty of equivariant eta functions near the origin. We also propose an equivariant version of the Cheeger-Chou index theorem on spaces with conelike singularities.
\end{abstract}

\section{INTRODUCTION}

Let $M$ be an odd-dimensional compact Riemannian spin manifold with a fixed spin structure, $D$ the Dirac operator on $M$. The $\eta$ function associated to $D$ is defined by [1]

$$
\eta(s, D)=\sum_{\lambda \neq 0}(\operatorname{sign} \lambda) \frac{\operatorname{dim} \Gamma\left(E_{\lambda}\right)}{|\lambda|^{s}},
$$

where $\lambda$ runs over the nonzero eigenvalues of $D$ and $\Gamma\left(E_{\lambda}\right)$ is the eigenspace of $\lambda$.

If $T: M \rightarrow M$ is an isometry preserving the orientation and spin structure and $\widetilde{d T} D=D \widetilde{d T}$, where $\widetilde{d T}: \Gamma(S(M)) \rightarrow \Gamma(S(M))$ is the lift of $d T: \Gamma(T M)$ $\rightarrow \Gamma(T M)$ to the spinors, then one can also define the equivariant $\eta$ function $[1,5]$ by

$$
\eta_{T}(s, D)=\sum_{\lambda \neq 0}(\operatorname{sign} \lambda) \frac{\left.\operatorname{Tr} \widetilde{d T}\right|_{\Gamma\left(E_{\lambda}\right)}}{|\lambda|^{s}}
$$

In the first two sections we prove some basic properties of equivariant $\eta$ functions and in $\S 3$, we point out that a slight modification of Bismut-Cheeger [3] yields an equivariant index theorem for spaces with conelike singularities.

Received by the editors April 28, 1989 and, in revised form, June 30, 1989.

1980 Mathematics Subject Classification (1985 Revision). Primary 58G10, 58G25.

Key words and phrases. Dirac operator, eta function, fixed points. 
Related work has also been done by J.-M. Bismut and J. Cheeger (unpublished).

\section{EQUiVARIANT $\eta$ FUNCTIONS}

It is clear that $(0.1)$ and $(0.2)$ are only defined when $\operatorname{Re}(s)$ is large enough. Then by analytic continuation to the whole complex plane, we obtain the meromorphic functions $\eta(s, D)$ and $\eta_{T}(s, D)$ on $\mathbf{C}$. Of particular interest is their regularity at $s=0$.

Theorem 1.1. For $\operatorname{Re}(s)$ large enough,

$$
\begin{aligned}
\eta_{T}(s, D) & =\frac{1}{\Gamma((s+1) / 2)} \int_{0}^{\infty} t^{(s-1) / 2} \operatorname{tr}\left(\widetilde{d T} D \exp \left(-t D^{2}\right)\right) d t \\
& =\frac{1}{\Gamma((s+1) / 2)} \int_{0}^{\infty} t^{(s-1) / 2} d t \int_{M} \operatorname{tr}\left(\widetilde{d T}_{x} D \exp \left(-t D^{2}\right)(x, T x)\right) d x,
\end{aligned}
$$

where $d x$ is the volume element associated to the metric, and $D \exp \left(-t D^{2}\right)(x, y)$ is the kernel of $D \exp \left(-t D^{2}\right)$.

Proof. For any $\lambda \neq 0$, let $\varphi_{\lambda_{1}}, \ldots, \varphi_{\lambda_{i(\lambda)}}$ be an orthonormal basis of $\Gamma\left(E_{\lambda}\right)$, then it is standard that

$$
D \exp \left(-t D^{2}\right)(x, y)=\sum_{\lambda \neq 0} \lambda e^{-t \lambda^{2}} \sum_{k=1}^{i(\lambda)} \varphi_{\lambda_{k}}(x)\left\langle\varphi_{\lambda_{k}}(y), \cdot\right\rangle .
$$

Let $\left\{e_{i}(x)\right\}$ be an orthonormal basis of $S_{x}$, then

$$
\widetilde{d T}_{x} D \exp \left(-t D^{2}\right)(x, T x) e_{i}(T x)=\sum_{\lambda} \lambda e^{-t \lambda^{2}} \sum_{k} \widetilde{d T} \varphi_{\lambda_{k}}(x)\left\langle\varphi_{\lambda_{k}}(T x), e_{i}(T x)\right\rangle .
$$

So

$$
\begin{aligned}
\operatorname{tr} \widetilde{d T} D \exp \left(-t D^{2}\right)(x, T x) \\
=\sum_{\lambda} \lambda e^{-t \lambda^{2}} \sum_{k} \sum_{i}\left\langle\widetilde{d T} \varphi_{\lambda_{k}}(x), e_{i}(T x)\right\rangle\left\langle\varphi_{\lambda_{k}}(T x), e_{i}(T x)\right\rangle \\
=\sum_{\lambda} \lambda e^{-t \lambda^{2}} \sum_{k}\left\langle\widetilde{d T} \varphi_{\lambda_{k}}(x), \varphi_{\lambda_{k}}(T x)\right\rangle
\end{aligned}
$$

and

$$
\begin{aligned}
\int_{M} \operatorname{tr} \widetilde{d T} D \exp \left(-t D^{2}\right)(x, T x) d x & =\sum_{\lambda} \lambda e^{-t \lambda^{2}} \int_{M} \sum_{k}\left\langle\widetilde{d T} \varphi_{\lambda_{k}}(x), \varphi_{\lambda_{k}}(T x)\right\rangle d x \\
& =\sum_{\lambda} \lambda e^{-t \lambda^{2}} \operatorname{tr}\left(\left.\widetilde{d T}\right|_{\Gamma\left(E_{\lambda}\right)}\right) .
\end{aligned}
$$


Thus

$$
\begin{aligned}
& \frac{1}{\Gamma((s+1) / 2)} \int_{0}^{\infty} t^{(s-1) / 2} \operatorname{tr}\left(\widetilde{d T} D \exp \left(-t D^{2}\right)\right) d t \\
& \quad=\left.\frac{1}{\Gamma((s+1) / 2)} \int_{0}^{\infty} t^{(s-1) / 2} \sum_{\lambda} \lambda e^{-t \lambda^{2}} \operatorname{tr} \widetilde{d T}\right|_{\Gamma\left(E_{\lambda}\right)} d t \\
& \quad=\sum_{\lambda \neq 0}(\operatorname{sign} \lambda) \frac{\left.\operatorname{tr} \widetilde{d T}\right|_{\Gamma\left(E_{\lambda}\right)}}{|\lambda|^{s}} .
\end{aligned}
$$

Corollary 1.4. If $T$ has no fixed points on $M$, then $\eta_{T}(s, D)$ is an entire function.

Proof. We introduce an auxiliary Grassmann variable $z$ as in [4]. Then we have

$$
\exp -t\left(D^{2}-z D\right)=\exp \left(-t D^{2}\right)+z t D \exp \left(-t D^{2}\right)
$$

so

$$
\exp \left(-t\left(D^{2}-z D\right)\right)(x, y)=\exp \left(-t D^{2}\right)(x, y)+z t D \exp \left(-t D^{2}\right)(x, y) .
$$

Since $T$ has no fixed points, $d(x, T x)>\delta$ for some constant $\delta>0$. By standard results for elliptic operators, there exist positive constants $C_{i} \quad(i=$ $1,2,3,4)$ such thai as $t \rightarrow 0^{+}$,

$$
\begin{gathered}
\left\|\exp \left(-t\left(D^{2}-z D\right)\right)(x, T x)\right\| \leq \frac{C_{1}}{t^{n / 2}} \exp \left(-C_{2} / t\right), \\
\left\|\exp \left(-t D^{2}\right)(x, T x)\right\| \leq \frac{C_{3}}{t^{n / 2}} \exp \left(-C_{4} / t\right)
\end{gathered}
$$

for all $x \in M$. Here we have assumed that $z$ has "norm" $\|z\|=1$. By (1.6)-(1.8), there are positive constants $C_{5}, C_{6}$ such that as $t \rightarrow 0^{+}$,

$$
\left\|D \exp \left(-t D^{2}\right)(x, T x)\right\| \leq \frac{C_{5}}{t^{n / 2+1}} \exp \left(-C_{6} / t\right) .
$$

Thus, $\forall s \in \mathbf{C}$

$$
\begin{aligned}
\lim _{t \rightarrow 0}\left|t^{(s-1) / 2} \operatorname{tr}\left(\widetilde{d T} D \exp \left(-t D^{2}\right)\right)\right| \\
\quad=\lim _{t \rightarrow 0}\left|t^{(s-1) / 2}\right| \cdot\left|\int_{M} \operatorname{tr} \widetilde{d T} D \exp \left(-t D^{2}\right)(x, T x) d x\right| \\
\quad \leq \lim _{t \rightarrow 0}\left|t^{(s-1) / 2}\right| \cdot C_{7} \frac{C_{5}}{t^{n / 2+1}} \exp \left(-C_{6} / t\right) \\
\quad=0
\end{aligned}
$$

where $C_{7}$ is a suitable positive constant.

Thus $\int_{0}^{\infty} t^{(s-1) / 2} \operatorname{tr}\left(\widetilde{d T} D \exp \left(-t D^{2}\right)\right) d t$ is an entire function. On the other hand, $1 / \Gamma((s+1) / 2)$ is also an entire function, (1.4) follows from Theorem 1.1 . 
Remark 1.11. In fact, (1.4) is a known result and can be proved directly without introducing the Grassmann variable $z$.

\section{REgULARITY NEAR $s=0$}

In this section, we prove the following theorem:

Theorem 2.1. $\eta_{T}(s, D)$ is holomorphic on $\operatorname{Re}(s)>-2$.

First note that it is sufficient to show that

$$
\left(\frac{1}{t}\right)^{1 / 2}\left|\operatorname{tr} \widetilde{d T} D \exp \left(-t D^{2}\right)\right| \leq C, \quad \text { as } t \rightarrow 0^{+}
$$

for some constant $C>0$. Because then the integration in (1.2) will be convergent absolutely and uniformly on the compact subsets in $\operatorname{Re}(s)>-2$, which implies the holomorphic property.

Now we begin to prove (2.2).

Since $T$ is an isometry, the fixed point set $F$ of $T$ consists of components $F_{1}, \ldots, F_{k}$, each of even codimension. If $U$ is an open neighborhood of $F$, then by the argument in the proof of (1.4), it is easy to see that

$$
\lim _{t \rightarrow 0}\left(\frac{1}{t}\right)^{1 / 2} \int_{M \backslash U} \operatorname{tr} \widetilde{d T} D \exp \left(-t D^{2}\right)(x, T x) d x=0,
$$

so

$$
\begin{aligned}
& \lim _{t \rightarrow 0}\left(\frac{1}{t}\right)^{1 / 2} \int_{M} \operatorname{tr} \widetilde{d T} D \exp \left(-t D^{2}\right)(x, T x) d x \\
& \quad=\lim _{t \rightarrow 0}\left(\frac{1}{t}\right)^{1 / 2} \int_{U} \operatorname{tr} \widetilde{d T} D \exp \left(-t D^{2}\right)(x, T x) d x .
\end{aligned}
$$

Thus we meet a local problem, and the situation is similar to what was considered in [8]. As in [8], we may assume $k=1$ and $\operatorname{codim} F=2 n^{\prime}$. Denote by $N(F)$ the normal bundle to $F$. We need only to prove that

$$
\lim _{t \rightarrow 0}\left(\frac{1}{t}\right)^{1 / 2}\left|\int_{F} \int_{N_{\xi}(\varepsilon)} \operatorname{tr} \widetilde{d T} D \exp \left(-t D^{2}\right)(x, d T x) d N_{\xi} d \xi\right| \leq C,
$$

for some constant $C>0$. Here $N_{\xi}(\varepsilon)=\left\{v \in N_{\xi}(F) \mid\|v\|<\varepsilon\right\}$, is a sufficiently small neighborhood of the origin in the normal space $N_{\xi}(F)$, and we use the local trivialization of $\exp N_{x}(\varepsilon)$ to $N_{x}(\varepsilon)$.

We choose an orthonormal basis and associated coordinates as in [8]. And we define as in $[9,8]$,

$$
\chi\left(x^{\alpha} D_{x}^{\beta} e^{\gamma}\right)=|\beta|-|\alpha|+|\gamma|,
$$

for $\alpha, \beta \in Z^{n}, \gamma \in\left(Z_{2}\right)^{n}$. We also define $\chi(z)=1$. Then we have

$$
\chi\left(z x^{\alpha} D_{x}^{\beta} e^{\gamma}\right)=1+|\beta|-|\alpha|+|\gamma| .
$$


Now as in [6], set

$$
h(x)=1+\frac{1}{2} z \sum_{i=1}^{n} x_{i} e_{i} .
$$

Then it is trivial to verify that

$$
\begin{aligned}
h e_{i} h^{-1} & =e_{i}+x_{i} z, \\
& =e_{i}+(\chi=0),
\end{aligned}
$$

and

$$
h\left(D^{2}-z D\right) h^{-1}=D^{2}+z u
$$

where $\chi(u) \leq 0$ and $u$ contains no $z$. Also write (2.10) as

$$
D^{2}-z D=h^{-1}\left(D^{2}+z u\right) h \text {. }
$$

Thus,

$$
\exp \left(-t\left(D^{2}-z D\right)\right)(x, y)=h^{-1}(x) \exp \left(-t\left(D^{2}+z u\right)\right)(x, y) h(y) .
$$

By (1.6) and $(2.11)^{\prime}$,

$$
\begin{aligned}
z t D \exp \left(-t D^{2}\right)(x, y)= & h^{-1}(x) \exp \left(-t\left(D^{2}+z u\right)\right)(x, y) h(y) \\
& -\exp \left(-t D^{2}\right)(x, y)
\end{aligned}
$$

Then

$z t \widetilde{d T} D \exp \left(-t D^{2}\right)(x, d T x)=\widetilde{d T} h^{-1}(x) \exp \left(-t\left(D^{2}+z u\right)\right)(x, d T x) h(d T x)$

$$
-\widetilde{d T} \exp \left(-t D^{2}\right)(x, d T x) \text {. }
$$

As in [9] (compare also with [4] or [7]), it is easy to see that

$$
\begin{gathered}
\exp \left(-t\left(D^{2}+z u\right)\right)(x, y)=\frac{e^{-d(x, y)^{2} / 4 t}}{(4 \pi t)^{n / 2}}\left(\sum_{i=0}^{[n / 2]+2}\left(U_{i}+z V_{i}\right) t^{i}+o\left(t^{[n / 2]+2}\right)\right) \\
\exp \left(-t D^{2}\right)(x, y)=\frac{e^{-d(x, y)^{2} / 4 t}}{(4 \pi t)^{n / 2}}\left(\sum_{i=0}^{[n / 2]+2} U_{i} t^{i}+o\left(t^{[n / 2]+2}\right)\right),
\end{gathered}
$$

where $\chi\left(U_{i}\right) \leq 2 i, \chi\left(V_{i}\right) \leq 2(i-1)$ and $U_{i}, V_{i}$ contain no $z$.

By (2.9) and (2.13)-(2.15), we get

$$
\begin{aligned}
& t \widetilde{d T D} \exp \left(-t D^{2}\right)(x, d T x) \\
& \begin{aligned}
=\frac{e^{-d(x, d T x)^{2} / 4 t}}{(4 \pi t)^{n / 2}} \widetilde{d T}\left[\left(\sum_{i}((d T-I) x)_{i} e_{i}\right)\right. & \cdot\left(\sum_{i=0}^{[n / 2]+2} U_{i} t^{i}+o\left(t^{[n / 2]+2}\right)\right) \\
& \left.+\left(\sum_{i=0}^{[n / 2]+2} W_{i} t^{i}+o\left(t^{[n / 2]+2}\right)\right)\right],
\end{aligned}
\end{aligned}
$$

where $\chi\left(W_{i}\right) \leq 2(i-1)$. 
Lemma 2.17. Suppose $i \leq[n / 2]+2$. If $W$ is an odd element and $\chi(W) \leq$ $2 i-2+2 n^{\prime}$, then

$$
\lim _{t \rightarrow 0}\left(\frac{1}{t}\right)^{3 / 2}\left|\int_{N_{\xi}(\varepsilon)} \frac{e^{-d(x, d T x)^{2} / 4 t}}{(4 \pi t)^{n / 2}} \operatorname{tr}(W(0 ; x)) t^{i} d x\right| \leq C_{1},
$$

for some constant $C_{1}>0$; where in the $W(y ; x), y$ stands for tangential coordinates and $x$ stands for normal coordinates.

Proof. We can assume that $W$ is a monomial, then it can be written as

$$
W=\varphi(0) x_{i_{1}} \cdots x_{i_{k}} \cdot e_{1} \cdots e_{n} .
$$

Here $e_{1} \cdots e_{n}$ all appear because otherwise, $\operatorname{tr} W=0$. Also note that we can assume that the $x_{i}$ 's in (2.19) are normal coordinates, for otherwise $\operatorname{tr} W(0 ; \cdot)=$ 0 .

(i) If $\chi(W)=2 i-2+2 n^{\prime}$, then $k=n+2-2 n^{\prime}-2 i$. By making the change of variables $x=t^{1 / 2} b$, we get

$$
\begin{aligned}
& \lim _{t \rightarrow 0}\left|\left(\frac{1}{t}\right)^{3 / 2} \int_{N_{\xi}(\varepsilon)} \frac{e^{-d(x, d T x)^{2} / 4 t}}{(4 \pi t)^{n / 2}} \operatorname{tr}(W) t^{i} d x\right| \\
& \quad \leq \lim _{t \rightarrow 0} \frac{C_{2}}{t^{3 / 2}}\left|\int_{N_{\xi}(\varepsilon / \sqrt{t})} \frac{e^{-\|(I-d T) b\|^{2} / 4}}{(4 \pi t)^{n / 2}} t^{n / 2+1-n^{\prime}-i} b_{i_{1}} \cdots b_{i_{k}} t^{i} t^{n^{\prime}} d b\right| \\
& \quad=0
\end{aligned}
$$

(because $k$ is odd).

(ii) If $\chi(W)<2 i-2+2 n^{\prime}$, then it is clear that

$$
\lim _{t \rightarrow 0}\left|\left(\frac{1}{t}\right)^{3 / 2} \int_{N_{\xi}(\varepsilon)} \frac{e^{-d(x, d T x)^{2} / 4 t}}{(4 \pi t)^{n / 2}} \operatorname{tr}(W) t^{i} d x\right| \leq C_{3} .
$$

Now consider the terms in (2.16). First note that the simple argument in [8] shows that

$$
\widetilde{d T}=\prod_{\alpha=n-2 n^{\prime}+1}^{n} e_{\alpha} \cdot f+g,
$$

where $\chi(f) \leq 0$ and $\chi(g) \leq 2 n^{\prime}-2$.

On the otherhand, $\left.d T\right|_{T F}=I d_{T F}$, so

$$
\sum_{i}((d T-I) x)_{i} e_{i}=\sum_{\alpha=n-2 n^{\prime}+1}^{n}((d T-I) x)_{\alpha} e_{\alpha} .
$$

By $(2.21),(2.20)$,

$$
\chi\left(\widetilde{d T} \cdot \sum_{i}((d T-I) x)_{i} e_{i}\right) \leq 2 n^{\prime}-2 .
$$


Thus, all the monomial terms in (2.16) satisfy the condition of Lemma 2.17. So we get

$$
\lim _{t \rightarrow 0}\left(\frac{1}{t}\right)^{1 / 2}\left|\int_{N_{\xi}(\varepsilon)} \operatorname{tr}\left(\widetilde{d T} D \exp \left(-t D^{2}\right)(x, d T x)\right) d x\right|<C_{4},
$$

for some constant $C_{4}>0$. By combining this with (2.5), (2.2), the proof of Theorem 2.1 is completed.

Remark 2.24. A more careful look at the proof allows us to write (2.2) in a more precise form:

Theorem 2.25. If $T: M \rightarrow M$ is an isometry and $F=\cup F_{i}$ is its fixed point set, then there exist smooth functions $\varphi_{i}$ on $F_{i}$ such that

$$
\operatorname{tr} \widetilde{d T} D \exp \left(-t D^{2}\right)=\sum_{i} \int_{F_{i}} \varphi_{i}(x) d x \cdot t^{1 / 2}+o\left(t^{1 / 2}\right), \quad t \searrow 0 .
$$

Remark 2.27. For $T=I d,(2.1)$ and (2.25) were obtained by Bismut-Freed [4] who improved an observation of Atiyah, Patodi, and Singer [1].

\section{AN INDEX THEOREM}

Now that we have established the basic properties of equivariant $\eta$ functions, an equivariant version of the index theorem of Cheeger and Chou (cf. [3]), can be obtained immediately by a slight modification of what was done in $\S 1$ of [3], where a new proof of the Cheeger-Chou theorem was given.

Here we just state the theorem and indicate briefly what should be modified. For notation and other details, we just refer to $[3, \S 1]$.

Let $Z$ denote a smooth connected compact manifold with smooth compact boundary $\partial Z$. Assume $Z$ has even dimension, is oriented and spin. Define

$$
C(\partial Z)=((0,1] \times \partial Z) \cup\{\delta\}
$$

and

$$
Z^{\prime}=Z \bigcup_{\partial Z} C(\partial Z)
$$

Introduce a metric $g^{Z^{\prime}, \varepsilon}$ on $Z^{\prime}$ as in [3].

Let $T: Z^{\prime} \rightarrow Z^{\prime}$ be an isometry, which is a product near $\partial Z$, i.e. it is a trivial extension of the isometry $\left.T\right|_{\partial Z}: \partial Z \rightarrow \partial Z$ to $(0,1+\varepsilon] \times \partial Z$ in a tubular neighborhood of $\partial Z$. Assume further more that $T$ has no fixed points on $\partial Z$. Let $D_{ \pm}^{\varepsilon}$ be the Dirac operator on $Z^{\prime}$ associated to $g^{Z^{\prime}, \varepsilon}$. Assume $\widetilde{d T} D_{ \pm}^{\varepsilon}=D_{ \pm}^{\varepsilon} \widetilde{d T}$. Then we can define the Lefschetz number

$$
L(T)=\left.\operatorname{tr} \widetilde{d T}\right|_{\mathrm{ker} D_{+}^{\varepsilon}}-\left.\operatorname{tr} \widetilde{d T}\right|_{\mathrm{ker} D_{-}^{\varepsilon}}
$$

Suppose as in [3] that

$$
\operatorname{ker} D^{\partial Z}=0
$$

then we can state the theorem as follows. 
Theorem 3.3. For $\varepsilon$ sufficiently small,

$$
\begin{aligned}
L(T)= & \sum_{i} \int_{F_{i}} \widehat{A}\left(T F_{i}\right)\left(P f(2 \sin (\Omega / 4 \pi+\sqrt{-1} \Theta / 2))\left(N\left(F_{i}\right)\right)\right)^{-1} \\
& -\frac{1}{2} \eta_{T}\left(0, D^{\partial Z}\right),
\end{aligned}
$$

where the $F_{i}$ are the components of the fixed point set of $T$ in $Z$, and the integrand is the standard density in Lefschetz fixed point formulas (cf., e.g. [8] or [2]).

The proof is almost the same as what was done in $[3, \S 1]$. The first point is to note that here we just use

$$
L(T)=\operatorname{tr}_{s} \widetilde{d T} \exp \left(-t\left(D^{\varepsilon}\right)^{2}\right)
$$

to replace the corresponding

$$
\text { ind } D_{+}^{\varepsilon}=\operatorname{tr}_{s}\left(\exp \left(-t\left(D^{\varepsilon}\right)^{2}\right)\right)
$$

in [3]. Then the proof is almost line by line the same as there. We have only to insert $\widetilde{d T}$ in all kernels, e.g. we write

$$
\widetilde{d T} P_{s}^{\varepsilon}((r, x),(r, T x))
$$

to replace

$$
P_{s}^{\varepsilon}((r, x),(r, x))
$$

in [3]. The only difference worth mentioning is that the equivariant replacement of (1.44) in [3] can be proved easily by using the fact that $T$ has no fixed points on $\partial Z$ and the proof of Corollary 1.4 in this note.

Details are omitted.

Also note that the index theorem of Atiyah-Patodi-Singer and Donnelly [5] for $G$-manifolds with boundary can also be deduced from (3.3) by a simple argument, similar to what was done in $\S 1, d$ ) of [3].

Remark 3.9. The condition (3.2) and the assumption that $T$ has no fixed points on the boundary $\partial Z$ are not essential. Indeed, this has been treated by J.-M. Bismut and J. Cheeger (unpublished). Here we will not go into the analytical details.

\section{ACKNOWLEDGMENTS}

The author wishes to thank Professor Yu Yanlin for helpful conversations and Professor Jeff Cheeger, Dr. Guoliang Yu and a referee for helpful comments on an earlier version of this paper.

\section{REFERENCES}

1. M. F. Atiyah, V. K. Patodi and I. M. Singer, Spectral asymmetry and Riemannian geometry, Math. Proc. Cambridge Philos. Soc. 77 (1975), 43-69; 78 (1975), 405-432; 79 (1976), 71-99.

2. N. Berline and M. Vergne, A computation of the equivariant index of the Dirac operators, Bull. Soc. Math. France 113 (1985), 305-345. 
3. J.-M. Bismut and J. Cheeger, Families index for manifolds with boundaries: superconnections and cones, J. Funct. Anal. (to appear).

4. J.-M. Bismut and D. S. Freed, The analysis of elliptic families II, Commun. Math. Phys. 107 (1986), 103-163.

5. H. Donnelly, Eta invariants for G-spaces, Indiana Univ. Math. J. 27 (1978), 889-918.

6. __ Local index theorem for families, Michigan Math. J. 35 (1988), 11-20.

7. E. Getzler, A short proof of the local Atiyah-Singer index theorem, Topology 25 (1986), 111117.

8. J. D. Lafferty, Y. L. Yu and W. P. Zhang, A direct geometric proof of Lefschetz fixed point formulas, preprint, 1988. (to appear in Trans. Amer. Math. Soc.)

9. Y. L. Yu, Local index theorem for Dirac operator, Acta Math. Sinica (New Series) 3 (1987), 152-169.

Nankai Institute of Mathematics, Weijin Road 94, Tianjin, 300071, People's Republic OF CHINA 